\title{
A SHORT INTRODUCTION TO DE BRANGES-ROVNYAK SPACES
}

\author{
DAN TIMOTIN
}

\section{Contents}

1. Introduction 1

2. Preliminaries 2

3. Introducing de Branges-Rovnyak spaces 3

3.1. Model spaces 3

3.2. Reproducing kernels 4

3.3. Contractively included subspaces 5

3.4. The complementary space 6

4. More about contractively included subspaces 8

5. Back to $\mathcal{H}(b) \quad 9$

5.1. Some properties of $\mathcal{H}(b)$; definition of $X_{b}$

5.2. Another representation of $\mathcal{H}(b)$ and $X_{b}$

5.3. The dichotomy extreme/nonextreme 11

6. The nonextreme case 11

7. The extreme case 13

8. $\mathcal{H}(b)$ as a model space 15

9. Further reading 17

Acknowledgements

References

\begin{abstract}
The notes provide a short introduction to de Branges-Rovnyak spaces. They cover some basic facts and are intended to give the reader a taste of the theory, providing sufficient motivation to make it interesting.
\end{abstract}

\section{INTRODUCTION}

The purpose of these notes is to provide a short introduction to de BrangesRovnyak spaces, that have been introduced in [6, 7], an area that has seen significant research activity in the last years. They are intended to give to a casual reader a taste of the theory, providing sufficient motivation and connections with other domains to make it, hopefully, interesting. There exist two comprehensive references on the subject: the older book of Sarason [22] that contains most of the basic facts, and the more recent monograph of Fricain and Mashreghi 14. The interested reader may study in depth the subject from there.

The author is partially supported by a grant of the Romanian National Authority for Scientific Research, CNCS UEFISCDI, project number PN-II-ID-PCE-2011-3-0119. 
The prerequisites are basic facts in operator theory on Hilbert space and in Hardy spaces. Many books contain them, but we have preferred to give as a comprehensive reference Nikolski's treaty [19, where all can be found (see the beginning of Section 2).

So the plan of the notes is the following. We introduce the de Branges-Rovnyak spaces as natural reproducing kernel spaces, generalizing the model spaces that appear prominently in the theory of contractions. The challenge here is to justify in a sufficient manner a rather exotic object of study, namely contractively included subspaces; we have considered the reproducing kernel approach as especially convenient. We give next some basic properties, following closely [22].

The dichotomy $b$ extreme/nonextreme appears soon. The general idea is that the extreme case has many features that are not far from the case of $b$ inner (the classical model spaces), while the nonextreme case is more exotic from this point of view.

Originally, the de Branges-Rovnyak spaces have been developed in view of model theory, that is, giving a universal model for certain class of operators on Hilbert space. They have been in the shade for a few decades, as the much more popular and well developed model theory of Sz-Nagy and Foias 24 has gained the upper hand. It is known to experts that the two theories are equivalent, and we thought that a justification of the study of de Branges-Rovnyak spaces should include some presentation of their role as model spaces. It turns out that this is easier done for the extreme case, and we have chosen to present this case at the end of the notes.

As noted above, the basic reference for de Branges-Rovnyak spaces, that has been frequently used in these notes, is the book of Sarason 22. Some simple unproved results appear in the text as exercises; for the others, references are indicated in the text at the relevant places, occasionally with a hint of the proof.

\section{Preliminaries}

A comprehensive reference for all facts in this section is [19], which has the advantage to contain both the necessary prerequisites from function theory (Part A, chapters 1-3) and from operator theory (part C, chapter 1).

If $H$ is a Hilbert space and $H^{\prime} \subset H$ a closed subspace, we will write $P_{H^{\prime}}$ for the orthogonal projection onto $H^{\prime}$. The space of bounded linear operators from $H_{1}$ to $H_{2}$ is denoted $\mathcal{B}\left(H_{1}, H_{2}\right)$; in case $H_{1}=H_{2}=H$ we write just $\mathcal{B}(H)$. If $T \in \mathcal{B}\left(H_{1}, H_{2}\right)$ is a contraction, we will denote by $D_{T}$ the selfadjoint operator $\left(I-T^{*} T\right)^{1 / 2}$ and by $\mathcal{D}_{T}$ the closed subspace $\overline{D_{T} H_{1}}=\operatorname{ker} T^{\perp}$. Thus $\mathcal{D}_{T} \subset H_{1}$ and $\mathcal{D}_{T^{*}} \subset H_{2}$. Obviously $D_{T} \mid \mathcal{D}_{T}$ is one-to-one.

Exercise 2.1. We have $T D_{T}=D_{T^{*}} T$. In particular, $T$ maps $\mathcal{D}_{T}$ into $\mathcal{D}_{T^{*}}$.

We may also consider the domain and/or the range of $D_{T}$ to be $\mathcal{D}_{T}$; by an abuse of notation all these operators will still be denoted by $D_{T}$. Note that the adjoint of $D_{T}: \mathcal{D}_{T} \rightarrow H_{1}$ is $D_{T}: H_{1} \rightarrow \mathcal{D}_{T}$.

Exercise 2.2. If $T \in \mathcal{B}(H)$ is a contraction, $H_{1} \subset H$ is a closed subset invariant by $T$, and we denote $T_{1}=T \mid H_{1}$, then $\mathcal{D}_{T_{1}}=\overline{P_{H_{1}} \mathcal{D}_{T}}$.

We denote by $L^{2}, L^{\infty}$ the Lebesgue spaces on the unit circle $\mathbb{T}$; we will also meet their closed subspaces $H^{2} \subset L^{2}$ and $H^{\infty} \subset L^{\infty}$ (the Hardy spaces). The corresponding norms will be denoted by $\|\cdot\|_{2}$ and $\|\cdot\|_{\infty}$ respectively. As usual, 
$H^{2}$ and $H^{\infty}$ can be identified with their analytic extension inside the unit disc $\mathbb{D}$. We assume known basic facts about inner and outer functions. We will write $P_{+}:=P_{H^{2}}$ (the orthogonal projection in $L^{2}$ ). The symbols $\mathbf{0}$ and $\mathbf{1}$ will denote the constant functions that take this value.

Each function $\phi$ in $L^{\infty}$ acts as multiplication on $L^{2}$; the corresponding operator will be denoted by $M_{\phi}$, and we have $\left\|M_{\phi}\right\|=\|\phi\|_{\infty}$. In particular, if $\phi(z)=z$, we will write $Z=M_{\phi}$. Actually, the commutant of $Z$ (the class of all operators $T$ on $L^{2}$ such that $Z T=T Z$ ) coincides precisely with the class of all $M_{\phi}$ for $\phi \in L^{\infty}$. (Obviously we have to define $\phi=T \mathbf{1} \in L^{2}$; a little work is needed to show that it is in $L^{\infty}$.)

The compression $P_{+} M_{\phi} P_{+}$restricted to the space $H^{2}$ is called the Toeplitz operator with symbol $\phi$ and is denoted by $T_{\phi}$. Again we have $\left\|T_{\phi}\right\|=\|\phi\|_{\infty}$; moreover, if $\phi \in H^{\infty}$, then $T_{\phi}$ is one-to-one (this is a consequence of the brothers Riesz Theorem: a function in $H^{2}$ is $\neq 0$ a.e.). In particular, if $\phi(z)=z$, we will write $S=T_{\phi}$; its adjoint $S^{*}$ acts as

$$
\left(S^{*} f\right)(z)=\frac{f(z)-f(0)}{z} .
$$

We have $T_{\phi}^{*}=T_{\bar{\phi}}$.

As noted above, the multiplication operators commute; this is in general not true for the Toeplitz operators.

Exercise 2.3. If $\phi \in H^{\infty}$, or $\psi \in \overline{H^{\infty}}$, then $T_{\psi} T_{\phi}=T_{\psi \phi}$.

If $k_{\lambda}(z)=\frac{1}{1-\lambda z}$ (a reproducing vector in $H^{2}$ - see more on reproducing kernels in Subsection 3.2 below), then for any $\phi \in H^{\infty}$ we have

$$
T_{\bar{\phi}} k_{\lambda}=\overline{\phi(\lambda)} k_{\lambda} .
$$

\section{Introducing De Branges-Rovnyak SPACES}

3.1. Model spaces. Beurling's theorem says that any subspace of $H^{2}$ invariant by $S$ is of the form $u H^{2}$, with $u$ an inner function.

Exercise 3.1. $S \mid u H^{2}$ is unitarily equivalent to $S$.

From some points of view, the orthogonal $\mathbf{K}_{u}=H^{2} \ominus u H^{2}$ is more interesting: it is a model space. It is invariant by $S^{*}$, but $S^{*} \mid \mathbf{K}_{u}$ may behave very differently. Actually, we know exactly how differently:

Theorem 3.2. If $T$ is a contraction on a Hilbert space $H$, then the following are equivalent:

(1) $I-T^{*} T$ and $I-T T^{*}$ have rank one and $T^{n}$ tends strongly to 0 .

(2) $T$ is unitarily equivalent to $S_{u}:=S^{*} \mid \mathbf{K}_{u} \in \mathcal{B}\left(\mathbf{K}_{u}\right)$ for some inner function $u$.

Theorem 3.2 is a particular case of the general Sz.-Nagy-Foias theory of contractions (see, for instance, the revised edition 24] of the original monography); one can find it also in [19].

So $S_{u}$ is a model operator for a certain class of contractions. We will meet in this course model operators for a more general class. 
3.2. Reproducing kernels. We introduce a larger class of spaces that include $\mathbf{K}_{u}$ for inner $u$; this will be done by means of reproducing kernels. A Hilbert space $\mathcal{R}$ of functions on a set $X$ is called a reproducing kernel space (RKS) if the evaluations at points of $X$ are continuous; we will always have $X=\mathbb{D}$. By Riesz's representation theorem it follows then that for each $\lambda \in \mathbb{D}$ there exists a function $\mathfrak{r}_{\lambda}^{\mathcal{R}} \in \mathcal{R}$, called the reproducing vector for $\lambda$, such that $f(\lambda)=\left\langle f, \mathcal{R}_{\lambda}^{\mathcal{R}}\right\rangle$. The function of two variables $\mathfrak{L}^{\mathcal{R}}(z, \lambda):=\mathfrak{l}_{\lambda}^{\mathcal{R}}(z)=\left\langle\mathfrak{l}_{\lambda}^{\mathcal{R}}, \mathfrak{l}_{z}^{\mathcal{R}}\right\rangle$ is called the reproducing kernel of the space $\mathcal{R}$. There is a one-to-one correspondence between RKS's and positive definite kernels (see for instance [1]).

Exercise 3.3. (1) If $\mathcal{R}$ is a RKS, and $\mathcal{R}_{1} \subset \mathcal{R}$ is a closed subspace, then $\mathcal{R}_{1}$ is also a RKS, and $\mathfrak{l}_{\lambda}^{\mathcal{R}_{1}}=P_{\mathcal{R}_{1}} \mathfrak{l}_{\lambda}^{\mathcal{R}}$.

(2) If $\mathcal{R}=\mathcal{R}_{1} \oplus \mathcal{R}_{2}$, then

$$
\mathfrak{L}^{\mathcal{R}}=\mathfrak{L}^{\mathcal{R}_{1}} \oplus \mathfrak{L}^{\mathcal{R}_{2}} .
$$

All three spaces discussed above have reproducing kernels, namely:

$$
\begin{gathered}
H^{2} \longrightarrow \frac{\frac{1}{1-\lambda z},}{u(\lambda) u(z)} \\
u H^{2} \longrightarrow \frac{1-\lambda z}{1-\overline{u(\lambda)} u(z)} \\
\mathbf{K}_{u} \longrightarrow \frac{1-\lambda z}{1-\lambda}
\end{gathered}
$$

and one can check that equality (3.1) is satisfied.

Our plan is to obtain RKSs with similar formulas, but replacing the inner function $u$ with an arbitrary function $b$ in the unit ball of $H^{\infty}$. That is, we want spaces with kernels $\frac{\overline{b(\lambda)} b(z)}{1-\bar{\lambda} z}$ and $\frac{1-\overline{b(\lambda)} b(z)}{1-\lambda z}$.

Of course it is not obvious that such RKSs exist. Then, if they exist, we want to identify them concretely, hoping to relate them to the familiar space $H^{2}$.

Things are simpler for the first kernel. Note first the next (general) exercise.

Exercise 3.4. If $\mathfrak{L}(z, \lambda)$ is a positive kernel on $X \times X$ and $\phi: X \rightarrow \mathbb{C}$, then $\phi(z) \overline{\phi(\lambda)} \mathfrak{L}(z, \lambda)$ is a positive kernel.

So $\frac{\overline{b(\lambda)} b(z)}{1-\lambda z}$ is the kernel of some space $\mathcal{R}$; but we would like to know it more concretely. The case $b=u$ inner suggests that a good candidate might be $b H^{2}$. Now, we already have a problem: if $b$ is a general function, $b H^{2}$ might not be closed in $H^{2}$, so it is not a genuine Hilbert space. But let us be brave and go on: we want

$$
\mathfrak{r}_{\lambda}^{\mathcal{R}}(z)=\overline{b(\lambda)} b(z) k_{\lambda} \text {. }
$$

Since the reproducing kernel property should be valid in $\mathcal{R}$, we must have, for any $f \in H^{2}$,

and therefore

$$
b(\lambda) f(\lambda)=\left\langle b f, \mathfrak{L}_{\lambda}^{\mathcal{R}}(z)\right\rangle_{\mathcal{R}}=b(\lambda)\left\langle b f, b k_{\lambda}\right\rangle_{\mathcal{R}}
$$

$$
f(\lambda)=\left\langle b f, b k_{\lambda}\right\rangle_{\mathcal{R}} .
$$

On the other hand, since $f \in H^{2}$, we have $f(\lambda)=\left\langle f, k_{z}\right\rangle_{H^{2}}$.

We have now arrived at the crucial point. If $b$ is inner, then $\left\langle b f, b k_{z}\right\rangle_{H^{2}}=$ $\left\langle f, k_{z}\right\rangle_{H^{2}}$ and everything is fine: the scalar product in $b H^{2}$ is the usual scalar 
product in $H^{2}$. But, in the general case, we have to define a different scalar product on $\mathcal{R}=b H^{2}$, by the formula

$$
\langle b f, b g\rangle_{\mathcal{R}}:=\langle f, g\rangle_{H^{2}} .
$$

This appears to solve the problem. Since $b f_{1}=b f_{2}$ implies $f_{1}=f_{2}$, formula (3.2) is easily shown to define a scalar product on $b H^{2}$. We will denote the corresponding Hilbert space by $\mathcal{M}(b)$. Let us summarize the results obtained.

Theorem 3.5. $\mathcal{M}(b)$, defined as $b H^{2}$ endowed with the scalar product (3.2), is a Hilbert space, which as a set is a linear subspace (in general not closed) of $H^{2}$. Its reproducing kernel is $\frac{\overline{b(\lambda)} b(z)}{1-\lambda z}$, and the inclusion $\iota: \mathcal{M}(b) \rightarrow H^{2}$ is a contraction. $\mathcal{M}(b)$ is invariant by $S$, and $S$ acts as an isometry on $\mathcal{M}(b)$.

Proof. From (3.2) it follows that the map $f \mapsto b f$ is isometric from $H^{2}$ onto $\mathcal{M}(b)$, whence $\mathcal{M}(b)$ is complete. The formula for the reproducing kernel has been proved (in fact, it lead to the definition of the space $\mathcal{M}(b)$ ). We have

$$
\|\iota(b f)\|_{H^{2}}=\|b f\|_{H^{2}} \leq\|f\|_{H^{2}}=\|b f\|_{\mathcal{M}(b)},
$$

and thus $\iota$ is a contraction.

Finally $\mathcal{M}(b)$ is invariant by $S$ since $z(b f)=b(z f)$, and

$$
\|z b f\|_{\mathcal{M}(b)}=\|b(z f)\|_{\mathcal{M}(b)}=\|z f\|_{H^{2}}=\|f\|_{H^{2}}=\|b f\|_{\mathcal{M}(b)},
$$

and thus the restriction of $S$ is an isometry.

This settles the case of the kernel $\frac{\overline{b(\lambda)} b(z)}{1-\lambda z}$. To discuss $\frac{1-\overline{b(\lambda)} b(z)}{1-\lambda z}$ is slightly more complicated, and a preliminary discussion is needed.

3.3. Contractively included subspaces. Let $T: E \rightarrow H$ be a bounded one-toone operator. Define on the image $T(E)$ a scalar product $\langle\cdot, \cdot\rangle^{\prime}$ by the formula

$$
\langle T \xi, T \eta\rangle^{\prime}:=\langle\xi, \eta\rangle_{E} .
$$

Then $T$ is a unitary operator from $E$ to $T(E)$ endowed with $\langle\cdot, \cdot\rangle^{\prime}$. The space obtained is complete; we will denote it by $M(T)$. The linear space $M(T)$ is contained as a set in $H$, and the inclusion is a contraction if and only if $T$ is a contraction. In this case the space $M(T)$ will be called a contractively included subspace of $H$, and the scalar product will be denoted $\langle\cdot, \cdot\rangle_{M(T)}$.

A slight modification is needed in case $T$ is not one-to-one; then (3.3) cannot be used directly since there $T \xi$ does not determine $\xi$ uniquely. We may however recapture this uniqueness and use (3.3) if we require that $\xi, \eta \in \operatorname{ker} T^{\perp}$; then $T$ becomes a unitary from $\operatorname{ker} T^{\perp}$ to $M(T)$.

In almost all cases in this course the corresponding contraction $T$ will be oneto-one, and thus we will apply directly (3.3). The only exception appears in Theorem 5.3. when we will use Lemma 3.6 below, with no direct reference to the scalar product.

We have already met a particular case of this notion: with the above notations, we have $\mathcal{M}(b)=M\left(T_{b}\right)$. Remember that, since $b \in H^{\infty}$, the operator $T_{b}$ is one-toone.

The following is a basic result that is used when we have to deal with two contractively embedded subspaces. It is essentially contained in [10].

Lemma 3.6. Suppose $T_{1}: E_{1} \rightarrow H, T_{2}: E_{2} \rightarrow H$ are two contractions. Then: 
(1) The space $M\left(T_{1}\right)$ is contained contractively in $M\left(T_{2}\right)$ (meaning that $M\left(T_{1}\right) \subset$ $M\left(T_{2}\right)$ and the inclusion is a contraction) if and only if $T_{1} T_{1}^{*} \leq T_{2} T_{2}^{*}$.

(2) The spaces $M\left(T_{1}\right)$ and $M\left(T_{2}\right)$ coincide as Hilbert spaces (that is, they are equal as sets, and the scalar product is the same) if and only if $T_{1} T_{1}^{*}=T_{2} T_{2}^{*}$.

(3) $T: H \rightarrow H$ acts contractively on $M\left(T_{1}\right)$ (meaning that $T\left(M\left(T_{1}\right)\right) \subset M\left(T_{1}\right)$ and $T \mid M\left(T_{1}\right)$ is a contraction) if and only if $T T_{1} T_{1}^{*} T^{*} \leq T_{1} T_{1}^{*}$.

It is worth at this point to note the following theorem of de Branges and Rovnyak [6], which is an analogue of Beurling's theorem.

Theorem 3.7. Suppose $X \subset H^{2}$ is a contractively included subspace of $H^{2}$. The following are equivalent

(1) $X$ is invariant by $S$ and the restriction $S \mid X$ is an isometry (in the norm of $X$ ).

(2) There exist a function $b$ in the unit ball of $H^{\infty}$, such that $X=\mathcal{M}(b)$.

The function $b$ is determined up to a multiplicative constant of modulus 1.

Suppose now that $H$ is a reproducing kernel Hilbert space, with kernel $\mathfrak{L}(z, \lambda)$. We may obtain for the reproducing vectors of $M(T)$ a formula similar to the particular case from the previous subsection.

Lemma 3.8. Suppose $T: E \rightarrow H$ is one-to-one. With the above notations, we have

$$
\mathfrak{l}_{\lambda}^{M(T)}=T T^{*} \mathfrak{l}_{\lambda}^{H}
$$

Proof. We have, using (3.3),

$$
\left\langle T f, T T^{*} \mathfrak{l}_{\lambda}^{H}\right\rangle_{M(T)}=\left\langle f, T^{*} \mathfrak{l}_{\lambda}^{H}\right\rangle_{H}=\left\langle T f, \mathfrak{l}_{\lambda}^{H}\right\rangle_{H}=(T f)(\lambda)=\left\langle T f, \mathfrak{l}_{\lambda}^{M(T)}\right\rangle_{M(T)},
$$

which proves the theorem.

In case $H=H^{2}, T=T_{b}$, we recapture the previous result: $M\left(T_{b}\right)=\mathcal{M}(b)$ and, using (2.2),

$$
\mathfrak{l}_{\lambda}^{\mathcal{M}(b)}=T_{b} T_{b}^{*} k_{\lambda}=b \overline{b(\lambda)} k_{\lambda}
$$

3.4. The complementary space. Remember that our current purpose is to find, if possible, an RKS with kernel $\frac{1-\overline{b(\lambda)} b(z)}{1-\lambda z}$. Let us note that

$$
\frac{1-\overline{b(\lambda)} b(z)}{1-\bar{\lambda} z}=\frac{1}{1-\bar{\lambda} z}-\frac{\overline{b(\lambda)} b(z)}{1-\bar{\lambda} z}=\mathfrak{L}^{H^{2}}(z, \lambda)-\mathfrak{L}^{\mathcal{M}(b)}(z, \lambda) .
$$

Can we obtain in the general case a formula for such a difference? The answer is positive.

Lemma 3.9. With the above notations,

$$
\mathfrak{L}^{H}(z, \lambda)-\mathfrak{L}^{M(T)}(z, \lambda)=\mathfrak{L}^{M\left(D_{T^{*}}\right)}(z, \lambda),
$$

where $D_{T^{*}}: \mathcal{D}_{T^{*}} \rightarrow H$ 
Proof. Using Lemma 3.8 we have

$$
\begin{aligned}
\mathfrak{L}^{H}(z, \lambda)-\mathfrak{L}^{M(T)}(z, \lambda) & =\left\langle\mathfrak{l}_{\lambda}^{H}, \mathfrak{l}_{z}^{H}\right\rangle_{H}-\left\langle\mathfrak{l}_{\lambda}^{M(T)}, \mathfrak{l}_{z}^{M(T)}\right\rangle_{M(T)} \\
& =\left\langle\mathfrak{l}_{\lambda}^{H}, \mathfrak{l}_{z}^{H}\right\rangle_{H}-\left\langle T T^{*} \mathfrak{l}_{\lambda}^{H)}, T T^{*} \mathfrak{l}_{z}^{H}\right\rangle_{M(T)} \\
& =\left\langle\mathfrak{l}_{\lambda}^{H}, \mathfrak{l}_{z}^{H}\right\rangle_{H}-\left\langle T^{*} \mathfrak{l}_{\lambda}^{H)}, T^{*} \mathfrak{l}_{z}^{H}\right\rangle_{E} \\
& =\left\langle\mathfrak{l}_{\lambda}^{H},\left(I-T T^{*}\right) \mathfrak{l}_{z}^{H}\right\rangle_{H}=\left\langle D_{T^{*}} \mathfrak{l}_{\lambda}^{H}, D_{T^{*}} \mathfrak{l}_{z}^{H}\right\rangle_{H} \\
& =\left\langle D_{T^{*}}^{2} \mathfrak{l}_{\lambda}^{H}, D_{T^{*}}^{2} \mathfrak{l}_{z}^{H}\right\rangle_{M\left(D_{T^{*}}\right)},
\end{aligned}
$$

(the last equality being a consequence of the fact that $D_{T^{*}}$ is one-to-one as an operator from $\mathcal{D}_{T^{*}}$ into $H$ ). Then Lemma 3.8 applied to $D_{T^{*}}$ instead of $T$, says that the last quantity is precisely $\mathfrak{L}^{M\left(D_{T^{*}}\right)}(z, \lambda)$.

Since $D_{T^{*}}$ is a contraction, the RKS corresponding to $\mathfrak{L}^{H}(z, \lambda)-\mathfrak{L}^{M(T)}(z, \lambda)$ is also a space contractively included in $H$; it is called the space complementary to $M(T)$ and will be denoted by $C(T)$.

Exercise 3.10. If $T$ is an isometry, then $M(T)$ is a usual subspace of $H$ (with the norm restricted), and $C(T)$ is its orthogonal complement.

If $x \in H$, then one can write

$$
x=T T^{*} x+D_{T^{*}}^{2} x .
$$

The first term in the right hand side is in $M(T)$, while the second is in $C(T)$. Moreover,

$$
\left\|T T^{*} x\right\|_{M(T)}^{2}=\left\|T^{*} x\right\|_{H}^{2}, \quad\left\|D_{T^{*}}^{2} x\right\|_{C(T)}^{2}=\left\|D_{T^{*}} x\right\|_{H}^{2},
$$

whence

$$
\|x\|^{2}=\left\|T T^{*} x\right\|_{M(T)}^{2}+\left\|D_{T^{*}}^{2} x\right\|_{C(T)}^{2} .
$$

In case $T$ is an isometry, $M(T)$ and $C(T)$ form an orthogonal decomposition of $H$, and (3.4) is the corresponding orthogonal decomposition of $H$. In the general case $M(T)$ and $C(T)$ may have a nonzero intersection, and so a decomposition $x=x_{1}+x_{2}$ with $x_{1} \in M(T), x_{2} \in C(T)$ is no more unique.

Exercise 3.11. If $T$ is a contraction, and $x=x_{1}+x_{2}$ with $x_{1} \in M(T)$, $x_{2} \in C(T)$, then

$$
\|x\|^{2} \leq\left\|x_{1}\right\|_{M(T)}^{2}+\left\|x_{2}\right\|_{C(T)}^{2},
$$

and equality implies $x_{1}=T T^{*} x, x_{2}=D_{T^{*}}^{2} x$.

At this point we have achieved our first purpose. Lemma 3.9 applied to the case $H=K=H^{2}$ and $T=T_{b}$ yields the identification of the reproducing kernel corresponding to $\frac{1-\overline{b(\lambda)} b(z)}{1-\lambda z}$.

Theorem 3.12. The RKS with kernel $\frac{1-\overline{b(\lambda)} b(z)}{1-\lambda z}$ is $M\left(D_{T_{b}^{*}}\right)$. It is a contractively included subspace of $H^{2}$ that will be denoted $\mathcal{H}(b)$ and called the de BrangesRovnyak space associated to the function $b$ in the inner ball of $H^{\infty}$.

As noted above, if $b=u$ is inner, then $\mathcal{H}(b)=\mathbf{K}_{u}$.

Exercise 3.13. (1) $H^{2}=\mathcal{H}(\mathbf{0})=\mathcal{M}(\mathbf{1})$.

(2) If $\|b\|_{\infty}<1$, then $\mathcal{H}(b)$ is a renormed version of $H^{2}$.

(3) If $\inf _{z \in \mathbb{D}}|b(z)|>0$, then $\mathcal{M}(b)$ is a renormed version of $H^{2}$. 
We will denote from now on $k_{\lambda}^{b}=\mathfrak{l}_{\lambda}^{\mathcal{H}(b)}=\frac{1-\overline{b(\lambda) b(z)}}{1-\bar{\lambda} z}$.

\section{More about Contractively inCluded subspaces}

Lemma 4.1. If $T: E \rightarrow H$ is a contraction, then:

(1) $\xi \in H$ belongs to $C(T)$ if and only if $T^{*} \xi \in C\left(T^{*}\right)$.

(2) If $\xi_{1}, \xi_{2} \in C(T)$, then

$$
\left\langle\xi_{1}, \xi_{2}\right\rangle_{C(T)}=\left\langle\xi_{1}, \xi_{2}\right\rangle_{H}+\left\langle T^{*} \xi_{1}, T^{*} \xi_{2}\right\rangle_{C\left(T^{*}\right)} .
$$

Proof. The inclusion $T^{*}(C(T)) \subset C\left(T^{*}\right)$ follows from the intertwining relation in Exercise 2.1. On the other hand, if $T^{*} \xi \in C\left(T^{*}\right)$, we have $T^{*} \xi=D_{T} \eta$ for some $\eta \in H$, and, using again Exercise 2.1.

$$
\xi=T T^{*} \xi+D_{T^{*}}^{2} \xi=T D_{T} \eta+D_{T^{*}}^{2} \xi=D_{T^{*}}\left(D_{T^{*}} \xi+T \eta\right),
$$

which shows that $\xi \in C(T)$.

To prove (2), write $\xi_{1}=D_{T^{*}} \eta_{1}, \xi_{2}=D_{T^{*}} \eta_{2}$, with $\eta_{1}, \eta_{2} \in \mathcal{D}_{T^{*}}$; then $T^{*} \eta_{1}, T^{*} \eta_{2} \in$ $\mathcal{D}_{T}$. Since both $D_{T}: \mathcal{D}_{T} \rightarrow H$ and $D_{T^{*}}: \mathcal{D}_{T^{*}} \rightarrow H$ are one-to-one, we have, using (3.3) and Exercise 2.1,

$$
\begin{aligned}
\left\langle\xi_{1}, \xi_{2}\right\rangle_{C(T)} & =\left\langle\eta_{1}, \eta_{2}\right\rangle=\left\langle D_{T^{*}} \eta_{1}, D_{T^{*}} \eta_{2}\right\rangle+\left\langle T^{*} \eta_{1}, T^{*} \eta_{2}\right\rangle \\
& =\left\langle\xi_{1}, \xi_{2}\right\rangle+\left\langle D_{T} T^{*} \eta_{1}, D_{T} T^{*} \eta_{2}\right\rangle_{C\left(T^{*}\right)} \\
& =\left\langle\xi_{1}, \xi_{2}\right\rangle+\left\langle T^{*} D_{T^{*}} \eta_{1}, T^{*} D_{T^{*}} \eta_{2}\right\rangle_{C\left(T^{*}\right)} \\
& =\left\langle\xi_{1}, \xi_{2}\right\rangle+\left\langle T^{*} \xi_{1}, T^{*} \xi_{2}\right\rangle_{C\left(T^{*}\right)} .
\end{aligned}
$$

There is a more direct way in which complementarity is related to orthogonality. If $T \in \mathcal{B}(E, H)$ is a contraction, we define the Julia operator $J(T): E \oplus \mathcal{D}_{T^{*}} \rightarrow$ $H \oplus \mathcal{D}_{T}$ by

$$
J(T)=\left(\begin{array}{cc}
T & D_{T^{*}} \\
D_{T} & -T^{*}
\end{array}\right) .
$$

Exercise 4.2. The Julia operator is unitary.

Lemma 4.3. Suppose $T: E \rightarrow H$ is one-to-one. Denote

$$
\mathcal{X}_{1}=J(T)(E \oplus\{0\}), \quad \mathcal{X}_{2}=\left(H \oplus \mathcal{D}_{T}\right) \ominus \mathcal{X}_{1}=J(T)\left(\{0\} \oplus \mathcal{D}_{T^{*}}\right),
$$

and by $P_{1}$ the projection of $H \oplus \mathcal{D}_{T}$ onto its first coordinate $H$. Then $P_{1} \mid \mathcal{X}_{1}$ is unitary from $\mathcal{X}_{1}$ onto $M(T)$, and $P_{1} \mid \mathcal{X}_{2}$ is unitary from $\mathcal{X}_{2}$ onto $C(T)$.

Proof. We have

$$
P_{1} \mathcal{X}_{1}=P_{1}\left(\left\{T x \oplus D_{T} x: x \in E\right\}\right)=\{T x: x \in E\}=M(T) .
$$

Moreover, if $x_{1} \in \mathcal{X}_{1}$, then $x_{1}=T x \oplus D_{T} x$ for some $x \in E$, and

$$
\left\|P_{1} x_{1}\right\|_{M(T)}=\|T x\|_{M(T)}=\|x\|_{E}=\|J(T)(x \oplus 0)\|=\left\|x_{1}\right\|,
$$

which proves the first part of the lemma.

Now $\mathcal{X}_{2}=J(T)\left(\{0\} \oplus \mathcal{D}_{T^{*}}\right)$, so

$$
P_{1} \mathcal{X}_{2}=P_{1}\left(\left\{D_{T^{*}} y \oplus-T^{*} y: y \in \mathcal{D}_{T^{*}}\right\}\right)=\left\{D_{T^{*}} y: y \in \mathcal{D}_{T^{*}}\right\}=C(T) .
$$

If $x_{2} \in \mathcal{X}_{2}$, then $x_{2}=D_{T^{*}} y \oplus-T^{*} y$ for some $y \in \mathcal{D}_{T^{*}}$, and

$$
\left\|P_{1} x_{2}\right\|_{C(T)}=\left\|D_{T^{*}} y\right\|_{C(T)}=\|y\|_{\mathcal{D}_{T^{*}}}=\|J(T)(0 \oplus y)\|=\left\|x_{2}\right\|,
$$

as required. 
We can view this result as saying that the orthogonal decomposition of $H \oplus \mathcal{D}_{T}$ as $\mathcal{X}_{1} \oplus \mathcal{X}_{2}$ is mapped by projecting onto the first coordinate into the complementary decomposition $H=M(T)+C(T)$ (which is not, in general, a direct sum). So the rather exotic definition of complementary spaces is in fact the projection of a more familiar geometric structure.

\section{5. ВАСK то $\mathcal{H}(b)$}

5.1. Some properties of $\mathcal{H}(b)$; definition of $X_{b}$. We denote $\mathcal{H}(\bar{b}):=\mathcal{H}\left(T_{\bar{b}}\right)$. Although our focus is on $\mathcal{H}(b)$, the space $\mathcal{H}(\bar{b})$ is a useful tool for its study.

Lemma 5.1. $\mathcal{H}(\bar{b})$ is contained contractively in $\mathcal{H}(b)$.

Proof. We have

$$
\begin{aligned}
T_{b} T_{\bar{b}} & =P_{+} M_{b} P_{+} M_{\bar{b}} P_{+}\left|H^{2} \leq P_{+} M_{b} M_{\bar{b}} P_{+}\right| H^{2} \\
& =P_{+} M_{\bar{b}} M_{b} P_{+}\left|H^{2}=P_{+} M_{\bar{b}} P_{+} M_{b} P_{+}\right| H^{2}=T_{\bar{b}} T_{b} .
\end{aligned}
$$

Therefore

$$
D_{T_{b}}^{2} \leq D_{T_{b}^{*}}^{2}
$$

whence Lemma 3.6(1) implies that $\mathcal{H}(\bar{b})$ is contained contractively in $\mathcal{H}(b)$.

Lemma 4.1 applied to the case $T=T_{b}$ yields the following result.

Lemma 5.2. If $h \in H^{2}$, then $h \in \mathcal{H}(b)$ if and only if $T_{\bar{b}} h \in \mathcal{H}(\bar{b})$. If $h_{1}, h_{2} \in \mathcal{H}(b)$, then

$$
\left\langle h_{1}, h_{2}\right\rangle_{\mathcal{H}(b)}=\left\langle h_{1}, h_{2}\right\rangle_{H^{2}}+\left\langle T_{\bar{b}} h_{1}, T_{\bar{b}} h_{2}\right\rangle_{\mathcal{H}(\bar{b})} .
$$

We now show that, similarly to model spaces, de Branges-Rovnyak spaces are invariant by adjoints of Toeplitz operators.

Theorem 5.3. If $\phi \in H^{\infty}$, then $\mathcal{H}(b)$ and $\mathcal{H}(\bar{b})$ are both invariant under $T_{\phi}^{*}=T_{\bar{\phi}}$, and the norm of this operator in each of these spaces is at most $\|\phi\|_{\infty}$.

Proof. We may assume that $\|\phi\|_{\infty} \leq 1$. By Lemma 3.6(3), in order to show that $T_{\bar{\phi}}$ acts as a contraction in $\mathcal{H}(\bar{b})$ we have to prove the inequality

$$
T_{\bar{\phi}}\left(I-T_{\bar{b}} T_{b}\right) T_{\phi} \leq I-T_{\bar{b}} T_{b},
$$

or

$$
\begin{aligned}
0 & \leq I-T_{\bar{b}} T_{b}-T_{\bar{\phi}}\left(I-T_{\bar{b}} T_{b}\right) T_{\phi}=I-T_{\bar{b}} T_{b}-T_{\bar{\phi}} T_{\phi}+T_{\bar{\phi}} T_{\bar{b}} T_{b} T_{\phi} \\
& =I-T_{|b|^{2}}-T_{|\phi|^{2}}+T_{|b|^{2}|\phi|^{2}}=T_{\left(1-|b|^{2}\right)\left(1-|\phi|^{2}\right) .}
\end{aligned}
$$

But the last operator is the compression to $H^{2}$ of $M_{\left(1-|b|^{2}\right)\left(1-|\phi|^{2}\right)}$, which is positive, since $\left(1-|b|^{2}\right)\left(1-|\phi|^{2}\right) \geq 0$.

This proves the statement for $\mathcal{H}(\bar{b})$. Take now $h \in \mathcal{H}(b)$. Lemma 5.2 implies that $T_{\bar{b}} h \in \mathcal{H}(\bar{b})$. By what has been just proved, $T_{\bar{b}} T_{\bar{\phi}} h=T_{\bar{\phi}} T_{\bar{b}} h \in \mathcal{H}(\bar{b})$, and then applying again Lemma 5.2 we obtain $T_{\bar{b}} h \in \mathcal{H}(b)$.

Finally, using (5.1) and the contractivity of $T_{\bar{\phi}}$ on $H^{2}$ as well as on $\mathcal{H}(\bar{b})$, we have

$$
\left\|T_{\bar{\phi}} h\right\|_{\mathcal{H}(b)}^{2}=\left\|T_{\bar{\phi}} h\right\|_{H^{2}}^{2}+\left\|T_{\bar{\phi}} T_{\bar{b}} h\right\|_{\mathcal{H}(\bar{b})}^{2} \leq\|h\|_{H^{2}}^{2}+\left\|T_{\bar{b}} h\right\|_{\mathcal{H}(\bar{b})}^{2}=\|h\|_{\mathcal{H}(b)}^{2},
$$

so $T_{\bar{\phi}}$ acts as a contraction in $\mathcal{H}(b)$. 
The most important case is obtained when $\phi(z)=z$. Theorem 5.3 says then that $\mathcal{H}(b)$ is invariant under $S^{*}$ and the restriction of $S^{*}$ is a contraction. We will denote by $X_{b}$ this restriction $S^{*} \mid \mathcal{H}(b)$.

Corollary 5.4. The function $S^{*} b$ is in $\mathcal{H}(b)$.

Proof. We have

$$
T_{\bar{b}} S^{*} b=S^{*} T_{\bar{b}} b=S^{*} T_{\bar{b}} T_{b} 1=-S^{*}\left(I-T_{\bar{b}} T_{b}\right) 1
$$

(for the last equality we have used the fact that $\left.S^{*} 1=0\right)$. Obviously $\left(I-T_{\bar{b}} T_{b}\right) 1 \in$ $\mathcal{H}(\bar{b})$, so Theorem 5.3 implies that $T_{\bar{b}} S^{*} b=-S^{*}\left(I-T_{\bar{b}} T_{b}\right) 1 \in \mathcal{H}(\bar{b})$. By Lemma 5.2 it follows that $S^{*} b \in \mathcal{H}(b)$.

Note that if $b \neq \mathbf{1}$, then $S^{*} b \neq \mathbf{0}$. Besides $S^{*} b$, we know as inhabitants of $\mathcal{H}(b)$ the reproducing vectors $k_{\lambda}^{b}$. Other elements may be obtained, for instance, by applying to these elements powers or functions of $X_{b}$.

Exercise 5.5. Show that, if $\lambda \in \mathbb{D}$, then

$$
\left(\left(I-\lambda X_{b}\right)^{-1}\left(S^{*} b\right)\right)(z)=\frac{b(z)-b(\lambda)}{z-\lambda} .
$$

Therefore the functions in the right hand side belong to $\mathcal{H}(b)$.

In general $b$ itself may not be in $\mathcal{H}(b)$; we will see later exactly when this happens. Let us also compute the adjoint of $X_{b}$.

Lemma 5.6. If $h \in \mathcal{H}(b)$, then

$$
X_{b}^{*} h=S h-\left\langle h, S^{*} b\right\rangle_{\mathcal{H}(b)} b .
$$

Proof. A computation shows that $X_{b} k_{\lambda}^{b}=S^{*} k_{\lambda}^{b}=\bar{\lambda} k_{\lambda}^{b}-\overline{b(\lambda)} S^{*} b$. Then, if $h \in \mathcal{H}(b)$ and $\lambda \in \mathbb{D}$, then

$$
\begin{aligned}
\left(X_{b}^{*} h\right)(\lambda) & =\left\langle X_{b}^{*} h, k_{\lambda}^{b}\right\rangle_{\mathcal{H}(b)}=\left\langle h, X_{b} k_{\lambda}^{b}\right\rangle_{\mathcal{H}(b)}=\lambda\left\langle h, k_{\lambda}^{b}\right\rangle_{\mathcal{H}(b)}-b(\lambda)\left\langle h, S^{*} b\right\rangle_{\mathcal{H}(b)} \\
& =\lambda h(\lambda)-\left\langle h, S^{*} b\right\rangle_{\mathcal{H}(b)} b(\lambda),
\end{aligned}
$$

which proves the lemma.

5.2. Another representation of $\mathcal{H}(b)$ and $X_{b}$. In the sequel of the course we will use the notation $\Delta=\left(1-|b|^{2}\right)^{1 / 2}$. The spaces $\overline{\Delta H^{2}}$ and $\overline{\Delta L^{2}}$ are closed subspaces of $L^{2}$ invariant with respect to $Z$. We will denote by $V_{\Delta}$ and $Z_{\Delta}$ the corresponding restrictions of $Z$.

Exercise 5.7. $V_{\Delta}$ is isometric, while $Z_{\Delta}$ is unitary.

The next result, a slight modification of Lemma 4.3. provides another representation of $\mathcal{H}(b)$.

Theorem 5.8. Suppose that $b$ is a function in the unit ball of $H^{\infty}$. Then:

(1) $S \oplus V_{\Delta}$ is an isometry on $H^{2} \oplus \overline{\Delta H^{2}}$.

(2) The space $\mathcal{K}_{b}:=\left(H^{2} \oplus \overline{\Delta H^{2}}\right) \ominus\left\{b h \oplus \Delta h: h \in H^{2}\right\}$ is a subspace of $H^{2} \oplus \overline{\Delta H^{2}}$ invariant with respect to $S^{*} \oplus V_{\Delta}^{*}$.

(3) The projection $P_{1}: H^{2} \oplus \overline{\Delta H^{2}} \rightarrow H^{2}$ on the first coordinate maps $\mathcal{K}_{b}$ unitarily onto $\mathcal{H}(b)$, and $P_{1}\left(S^{*} \oplus V_{\Delta}^{*}\right)=X_{b} P_{1}$. 
Proof. The proof of (1) is immediate. Also, the map $h \mapsto b h \oplus \Delta h$ is an isometry of $H^{2}$ onto $\left\{b h \oplus \Delta h: h \in H^{2}\right\}$, which is therefore a closed subspace. Since $\left(S \oplus V_{\Delta}\right)(b h \oplus \Delta h)=b(z h) \oplus \Delta(z h)$, it is immediate that $\left\{b h \oplus \Delta h: h \in H^{2}\right\}$ is invariant by $S \oplus V_{\Delta}$, whence its orthogonal $\mathcal{K}$ is invariant by $S^{*} \oplus V_{\Delta}^{*}$; thus (2) is proved.

To prove (3), let us apply Lemma 4.3 to the case $T=T_{b}$, when $C(T)=\mathcal{H}(b)$. It says that, if $\mathcal{X}_{2}=\left(H^{2} \oplus \mathcal{D}_{T_{b}}\right) \ominus\left\{T_{b} h \oplus D_{T_{b}} h\right\}$, then the projection onto the first coordinate maps $\mathcal{X}_{2}$ unitarily onto $\mathcal{H}(b)$. Since, for any $h \in H^{2}$,

$\left\|D_{T_{b}} h\right\|^{2}=\|h\|^{2}-\|b h\|^{2}=\frac{1}{2 \pi} \int_{-\pi}^{\pi}\left|h\left(e^{i t}\right)\right|^{2} d t-\frac{1}{2 \pi} \int_{-\pi}^{\pi}\left|b\left(e^{i t}\right) h\left(e^{i t}\right)\right|^{2} d t=\|\Delta h\|^{2}$,

the map $D_{T_{b}} h \mapsto \Delta h$ extends to a unitary $U$ from $\mathcal{D}_{T_{b}}$ onto the closure of $\overline{\Delta H^{2}}$. Then $I_{H^{2}} \oplus U$ maps unitarily $H^{2} \oplus \mathcal{D}_{T_{b}}$ onto $H^{2} \oplus \overline{\Delta H^{2}}, \mathcal{X}_{1}$ onto $\left\{b h \oplus \Delta h: h \in H^{2}\right\}$, $\mathcal{X}_{2}$ onto $\mathcal{K}_{b}$, and it commutes with the projection on the first coordinate. Therefore $P_{1}$ maps $\mathcal{K}_{b}$ unitarily onto $\mathcal{H}(b)$, and

$$
P_{1}\left(S^{*} \oplus V_{\Delta}^{*}\right)\left|\mathcal{K}_{b}=P_{1}\left(S^{*} \oplus V_{\Delta}^{*}\right) P_{1}\right| \mathcal{K}_{b}=\left(S^{*} \oplus 0\right) P_{1} \mid \mathcal{K}_{b}=\left(S^{*} \mid \mathcal{H}(b)\right) P_{1}=X_{b} P_{1} .
$$

5.3. The dichotomy extreme/nonextreme. The study of the spaces $\mathcal{H}(b)$ splits further into two mutually exclusive cases: when $b$ is an extreme point of the unit ball of $H^{\infty}$ and when it is not. The first case includes $b=u$ inner, and thus will be more closely related to model spaces, while the second includes the case $\|b\|<1$, and thus there will be properties similar to the whole of $H^{2}$. Actually, we will not use extremality directly, but rather through one of the equivalent characterizations given by the next lemma (for which again [19] can be used as a reference).

Lemma 5.9. If $h$ is a function in the unit ball of $H^{\infty}$, then the following are equivalent:

(1) $b$ is extreme.

(2) $\frac{1}{2 \pi} \int_{-\pi}^{\pi} \log \Delta\left(e^{i t}\right) d t=-\infty$.

(3) $\overline{\Delta H^{2}}=\overline{\Delta L^{2}}$.

\section{The noneXtreme CASE}

When $\frac{1}{2 \pi} \int_{-\pi}^{\pi} \log \Delta\left(e^{i t}\right) d t>-\infty$, there exists a uniquely defined outer function $a$ with $|a|=\Delta$ and $a(0)>0$; thus $|a|^{2}+|b|^{2}=1$. This function is a basic tool in the theory of $\mathcal{H}(b)$ in the nonextreme case. Since (see Exercise 2.3)

$$
T_{\bar{a}} T_{a}=T_{\bar{a} a}=I-T_{\bar{b} b}=I-T_{\bar{b}} T_{b}
$$

Lemma 3.6 (2) implies that $\mathcal{H}(\bar{b})=\mathcal{M}(\bar{a})$.

Exercise 6.1. If $a$ is an outer function, then $\operatorname{ker} T_{\bar{a}}=\{0\}$.

We can apply Lemma 5.2 to the current situation.

Lemma 6.2. (1) We have $h \in \mathcal{H}(b)$ if and only if $T_{\bar{b}} h \in \mathcal{M}(\bar{a})$; when this happens there is a unique (by Exercise 6.1) function $h^{+} \in H^{2}$ such that $T_{\bar{b}} h=T_{\bar{a}} h^{+}$.

(2) If $h_{1}, h_{2} \in \mathcal{H}(b)$, then

$$
\left\langle h_{1}, h_{2}\right\rangle_{\mathcal{H}(b)}=\left\langle h_{1}, h_{2}\right\rangle_{H^{2}}+\left\langle h_{1}^{+}, h_{2}^{+}\right\rangle_{H^{2}} .
$$

(3) If $h \in \mathcal{H}(b)$ and $\phi \in H^{\infty}$, then $\left(T_{\bar{\phi}} h\right)^{+}=T_{\bar{\phi}} h^{+}$. 
Proof. (1) is a consequence of Lemma 5.2 and the equality $\mathcal{H}(\bar{b})=\mathcal{M}(\bar{a})$; the uniqueness of $h^{+}$follows from Exercise 6.1.

The formula for the scalar product in Lemma 5.2 becomes

$$
\left\langle h_{1}, h_{2}\right\rangle_{\mathcal{H}(b)}=\left\langle h_{1}, h_{2}\right\rangle_{H^{2}}+\left\langle T_{\bar{a}} h_{1}^{+}, T_{\bar{a}} h_{2}^{+}\right\rangle_{\mathcal{H}(\bar{a})}=\left\langle h_{1}^{+}, h_{2}^{+}\right\rangle_{H^{2}},
$$

the last equality being a consequence of the fact that $T_{\bar{a}}$ is one-to-one. This proves (2).

Finally,

proving (3).

$$
T_{\bar{b}} T_{\bar{\phi}} h=T_{\bar{\phi}} T_{\bar{b}} h=T_{\bar{\phi}} T_{\bar{a}} h^{+}=T_{\bar{a}} T_{\bar{\phi}} h^{+},
$$

We gather in a theorem some properties of $\mathcal{H}(b)$ for $b$ nonextreme.

Theorem 6.3. Suppose $b$ is nonextreme.

(1) The polynomials belong to $\mathcal{M}(\bar{a})$ and are dense in $\mathcal{M}(\bar{a})$.

(2) $\mathcal{M}(\bar{a})$ is dense in $\mathcal{H}(b)$.

(3) The polynomials are dense in $\mathcal{H}(b)$.

(4) The function $b$ is in $\mathcal{H}(b)$, and $\|b\|_{\mathcal{H}(b)}^{2}=|a(0)|^{-2}-1$.

(5) The space $\mathcal{H}(b)$ is invariant by the unilateral shift $S$.

Proof. By checking the action on monomials, it is immediate that the space $\mathcal{P}_{n}$ of polynomials of degree less or equal to $n$ is invariant by $T_{\bar{a}}$. But $T_{\bar{a}}$ is one-to-one, and so $T_{\bar{a}} \mid \mathcal{P}_{n}$ is also onto. So all polynomials belong to the image of $T_{\bar{a}}$, which is $\mathcal{M}(\bar{a})$. Moreover, since $T_{\bar{a}}$ is one-to-one, it is unitary as an operator from $H^{2}$ to $\mathcal{M}(\bar{a})$. Then the image of all polynomials, which form a dense set in $H^{2}$, is a dense set in $\mathcal{M}(\bar{a})$ which proves (1).

Suppose that $h \in \mathcal{H}(b)$ is orthogonal in $\mathcal{H}(b)$ to all $\mathcal{M}(\bar{a})$. In particular, $h$ is orthogonal to $T_{\bar{a}} S^{* n} h$ for every $n \geq 0$. By Lemma $6.2(3),\left(T_{\bar{a}} S^{* n} h\right)^{+}=T_{\bar{a}} S^{* n} h^{+}$; applying then 6.2(2), we have, for any $n \geq 0$,

$$
\begin{aligned}
0 & =\left\langle h, T_{\bar{a}} S^{*} n h\right\rangle_{\mathcal{H}(b)} \\
& =\left\langle h, T_{\bar{a}} S^{*} n h\right\rangle_{H^{2}}+\left\langle h^{+}, T_{\bar{a}} S^{*} n h^{+}\right\rangle_{H^{2}} \\
& =\frac{1}{2 \pi} \int_{-\pi}^{\pi} a\left(e^{i t}\right)\left(\left|h\left(e^{i t}\right)\right|^{2}+\left|h^{+}\left(e^{i t}\right)\right|^{2}\right) e^{i n t} d t .
\end{aligned}
$$

Therefore, the function $a\left(|h|^{2}+\left|h^{+}\right|^{2}\right)$ belongs to $H_{0}^{1}$. A classical fact about outer functions (see, for instance, [19]) implies that we also have $|h|^{2}+\left|h^{+}\right|^{2} \in H_{0}^{1}$. But the only real-valued function in $H_{0}^{1}$ is the zero function, so $h=0$, which proves (2). Obviously, (1) and (2) imply (3).

We have

$$
T_{\bar{b}} b=P_{+}(\bar{b} b)=P_{+}(1-\bar{a} a)=T_{\bar{a}}(1 / \overline{a(0)}-a) .
$$

By Lemma 6.2 it follows that $b \in \mathcal{H}(b)$ and $b^{+}=1 / \overline{a(0)}-a$; moreover,

$$
\begin{aligned}
\|b\|_{\mathcal{H}(b)}^{2} & =\|b\|_{H^{2}}^{2}+\|1 \overline{a(0)}-a\|_{H^{2}}^{2} \\
& =\|b\|_{H^{2}}^{2}+\|a-a(0)\|_{H^{2}}^{2}+\|1 \overline{a(0)}-a(0)\|_{H^{2}}^{2} \\
& =\|b\|_{H^{2}}^{2}+\|a\|_{H^{2}}^{2}-|a(0)|^{2}+|a(0)|^{-2}+|a(0)|^{2}-2 \\
& =|a(0)|^{-2}-1,
\end{aligned}
$$

which proves (4).

Finally, Lemma 5.6 together with (4) prove the invariance of $\mathcal{H}(b)$ to $S$. 


\section{The EXTREMe CASE}

We point out first some differences with respect to the nonextreme case.

Theorem 7.1. Suppose $b$ is extreme. Then:

(1) The function $b$ does not belong to $\mathcal{H}(b)$.

(2) If $b \neq \mathbf{1}$, then $\mathcal{H}(b)$ is not invariant by $S$.

Proof. Suppose $b \in \mathcal{H}(b)$. By Theorem 5 .8, it follows that there exists $\psi \in \overline{\Delta H^{2}} \subset$ $L^{2}$, such that $b \oplus \psi \perp\left\{b h \oplus \Delta h: h \in H^{2}\right\}$. So

$$
\langle b \oplus \psi, b h \oplus \Delta h\rangle=0, \text { for all } h \in H^{2},
$$

which is equivalent to $|b|^{2}+\Delta \psi \in \overline{H_{0}^{2}}$. This is equivalent to $1-\Delta^{2}+\Delta \psi \in \overline{H_{0}^{2}}$, whence $f:=\Delta^{2}-\Delta \bar{\psi}$ is a nonzero (note that its zeroth Fourier coefficient is 1 ) function in $H^{2}$. Thus $\Delta^{-1} f=\Delta-\bar{\psi} \in L^{2}$, or $\Delta^{-2}|f|^{2} \in L^{1}$.

We assert that this is not possible. Indeed,

$$
\Delta^{-2}|f|^{2} \geq \log \left(\Delta^{-2}|f|^{2}\right)=2 \log |f|-2 \log \Delta .
$$

Integrating, we obtain

$$
\frac{1}{2 \pi} \int \Delta^{-2}\left(e^{i t}\right)\left|f\left(e^{i t}\right)\right|^{2} d t \geq 2 \frac{1}{2 \pi} \int \log \left|f\left(e^{i t}\right)\right| d t+2 \frac{1}{2 \pi} \int\left(-\log \Delta\left(e^{i t}\right)\right) d t,
$$

which cannot be true, since the first two integrals are finite, while the third is infinite by Lemma 5.9.

We have thus proved (1). Then (2) follows from Lemma 5.6, which can be restated as

$$
\left\langle h, S^{*} b_{\mathcal{H}(b)}\right\rangle b=S h-X_{b}^{*} h .
$$

So, if we choose $h$ not orthogonal (in $\mathcal{H}(b)$ ) to $S^{*} b$ (in particular, $h=S^{*} b$ ), we must have $S h \notin \mathcal{H}(b)$.

In the sequel we will use the geometrical representation of $\mathcal{H}(b)$ given by Theorem 5.8. Using Lemma 5.9. we may replace in its statement $\overline{\Delta H^{2}}$ by $\overline{\Delta L^{2}}$.

Denote $\tilde{b}(z)=\overline{b(\bar{z})}$, and $\tilde{\Delta}=\left(1-|\tilde{b}|^{2}\right)^{1 / 2}$. The map $f \mapsto \tilde{f}$ is a unitary involution that maps $L^{2}$ onto $L^{2}, H^{2}$ onto $H^{2}$, and $\overline{\tilde{\Delta} L^{2}}$ onto $\overline{\Delta L^{2}}$.

Exercise 7.2. $b$ is extreme if and only if $\tilde{b}$ is extreme.

Theorem 7.3. Suppose $b$ is extreme. Define

$$
\Omega: L^{2} \oplus \overline{\tilde{\Delta} L^{2}} \rightarrow L^{2} \oplus \overline{\Delta L^{2}}
$$

by the formula

$$
\Omega(f \oplus g)=(b(z) \bar{z} f(\bar{z})+\Delta \bar{z} g(\bar{z})) \oplus(\Delta \bar{z} f(\bar{z})-\bar{b} \bar{z} g(\bar{z})) .
$$

Then $\Omega$ is unitary, it maps $\mathcal{K}_{\tilde{b}}$ onto $\mathcal{K}_{b}$, and

$$
\Omega X_{\tilde{b}}=X_{b}^{*} \Omega
$$


Proof. $\Omega$ acts as the unitary $f \oplus g \mapsto \bar{z} f(\bar{z}) \oplus \bar{z} g(\bar{z})$ followed by the unitary $J\left(M_{b}\right)$, so it is unitary. We have

$$
\begin{aligned}
& \mathcal{K}_{b}=\left(L^{2} \oplus \overline{\Delta L^{2}}\right) \ominus\left[\left(H_{-}^{2} \oplus\{0\}\right) \oplus\left(\left\{b f \oplus \Delta f: f \in H^{2}\right\}\right)\right], \\
& \mathcal{K}_{\tilde{b}}=\left(L^{2} \oplus \overline{\tilde{\Delta} L^{2}}\right) \ominus\left[\left(H_{-}^{2} \oplus\{0\}\right) \oplus\left(\left\{\tilde{b} f \oplus \tilde{\Delta} f: f \in H^{2}\right\}\right)\right] .
\end{aligned}
$$

If $f \in H_{-}^{2}$, then

$$
\Omega(f \oplus 0)=b \bar{z} f(\bar{z}) \oplus \Delta \bar{z} f(\bar{z}) .
$$

But the map $f \mapsto \bar{z} f(\bar{z})$ is a unitary from $H_{-}^{2}$ onto $H^{2}$, whence it follows that $\Omega\left(H_{-}^{2} \oplus\{0\}\right)=\left\{b f \oplus \Delta f: f \in H^{2}\right\}$. Similarly we obtain $\Omega\left(\left\{b f \oplus \tilde{\Delta} f: f \in H^{2}\right\}\right)=$ $H_{-}^{2} \oplus\{0\}$. Therefore

$$
\Omega\left(\left(H_{-}^{2} \oplus\{0\}\right) \oplus\left(\left\{\tilde{b} f \oplus \tilde{\Delta} f: f \in H^{2}\right\}\right)\right)=\left(H_{-}^{2} \oplus\{0\}\right) \oplus\left(\left\{b f \oplus \Delta f: f \in H^{2}\right\}\right),
$$

whence $\Omega\left(\mathcal{K}_{\tilde{b}}\right)=\Omega\left(\mathcal{K}_{b}\right)$.

Finally, we have $X_{b}=P_{\mathcal{K}_{b}}\left(Z^{*} \oplus Z_{\Delta}^{*}\right) P_{\mathcal{K}_{b}} \mid \mathcal{K}_{b}$ and $X_{\tilde{b}}=P_{\mathcal{K}_{\tilde{b}}}\left(Z^{*} \oplus Z_{\tilde{\Delta}}^{*}\right) P_{\mathcal{K}_{\tilde{b}}} \mid \mathcal{K}_{\tilde{b}}$. But $\Omega\left(\mathcal{K}_{\tilde{b}}\right)=\Omega\left(\mathcal{K}_{b}\right)$ implies $P_{\mathcal{K}_{\tilde{b}}}=\Omega^{*} P_{\mathcal{K}_{b}} \Omega$. Therefore

$$
\begin{aligned}
\Omega X_{\tilde{b}} & =\Omega P_{\mathcal{K}_{\tilde{b}}}\left(Z^{*} \oplus Z_{\tilde{\Delta}}^{*}\right) P_{\mathcal{K}_{\tilde{b}}}\left|\mathcal{K}_{\tilde{b}}=\Omega \Omega^{*} P_{\mathcal{K}_{b}} \Omega\left(Z^{*} \oplus Z_{\tilde{\Delta}}^{*}\right) \Omega^{*} P_{\mathcal{K}_{b}} \Omega\right| \mathcal{K}_{\tilde{b}} \\
& =P_{\mathcal{K}_{b}} \Omega\left(Z^{*} \oplus Z_{\tilde{\Delta}}^{*}\right) \Omega^{*} P_{\mathcal{K}_{b}} \Omega \mid \mathcal{K}_{\tilde{b}} .
\end{aligned}
$$

But one checks easily that $\Omega\left(Z^{*} \oplus Z_{\tilde{\Delta}}^{*}\right) \Omega^{*}=Z \oplus Z_{\Delta}$, and therefore

$$
\begin{aligned}
P_{\mathcal{K}_{b}} \Omega\left(Z^{*} \oplus Z_{\tilde{\Delta}}^{*}\right) \Omega^{*} P_{\mathcal{K}_{b}} \Omega \mid \mathcal{K}_{\tilde{b}} & =P_{\mathcal{K}_{b}}\left(Z \oplus Z_{\Delta}\right) P_{\mathcal{K}_{b}} \Omega \mid \mathcal{K}_{\tilde{b}} \\
& =\left(P_{\mathcal{K}_{b}}\left(Z^{*} \oplus Z_{\Delta}^{*}\right) P_{\mathcal{K}_{b}}\right)^{*} \Omega \mid \mathcal{K}_{\tilde{b}}=X_{b}^{*} \Omega
\end{aligned}
$$

which ends the proof of the theorem.

We note that in case $b$ is nonextreme $X_{b}^{*}$ is never unitarily equivalent to $X_{\tilde{b}}$.

Theorem 7.4. Suppose $b$ is extreme. Then $\operatorname{dim} \mathcal{D}_{X_{b}}=\operatorname{dim} \mathcal{D}_{X_{b}^{*}}=1$, and there exists no subspace of $\mathcal{H}_{b}$ invariant by $X$ and such that its restriction therein is an isometry.

Proof. From Theorem 5.8(3) it follows that we may prove the properties for the restriction $S^{*} \oplus Z_{\Delta}^{*} \mid \mathcal{K}_{b}$. Since $S^{*}$ acts isometrically on $H_{0}^{2}$ and $Z_{\Delta}^{*}$ is unitary, we have $\mathcal{D}_{S^{*} \oplus Z_{\Delta}^{*}}=\mathbb{C}(1 \oplus 0)$. By Exercise 2.2, $\mathcal{D}_{X_{b}}=\mathbb{C} P_{\mathcal{K}_{b}}(1 \oplus 0)$. But $P_{\mathcal{K}_{b}}(1 \oplus 0) \neq 0$; indeed, otherwise we would have $1 \oplus 0=b h \oplus \Delta h$ for some $h \in H^{2}$; since $h \neq 0$ a.e., this would imply $\Delta=0$ a.e., or $b$ inner, which is impossible if $1=b h$. Therefore $\operatorname{dim} \mathcal{D}_{X}=1$.

Applying the same argument to $\tilde{b}$ and using (7.1), it follows that $\operatorname{dim} \mathcal{D}_{X^{*}}=1$.

Finally, suppose $\mathcal{Y} \subset \mathcal{K}$ is a closed subspace on which $X_{b}$ acts isometrically, and $h \oplus g \in \mathcal{Y}$, we have, for any $n \geq 0$,

$\|h\|^{2}+\|g\|^{2}=\|h \oplus g\|^{2}=\left\|S^{* n} h \oplus Z_{\Delta}^{* n} g\right\|^{2}=\left\|S^{*} h^{n}\right\|^{2}+\left\|Z_{\Delta}^{* n} g\right\|^{2} \rightarrow\left\|Z_{\Delta}^{* n} g\right\|^{2}=\|g\|^{2}$, whence $h=0$. But then we must have $0 \oplus g \perp b f \oplus \Delta f$ for all $f \in H^{2}$, or $g \perp \overline{\Delta H^{2}}=\overline{\Delta L^{2}}$. Since, on the other hand, $g \in \overline{\Delta L^{2}}$, it follows that $g=0$, which ends the proof of the theorem. 


\section{8. $\mathcal{H}(b)$ AS A MODEL SPACE}

The purpose of this section is to prove the converse of Theorem 7.4. This will show that in the extreme case the de Branges-Rovnyak spaces are model spaces for a large class of operators. The theorem below (as well as its proof) is in fact a particular case of the much more general analysis of contractions done in the Sz.Nagy-Foias theory (see 24]). Here we have adapted the argument to a "minimal" self-contained form.

Theorem 8.1. Suppose $T \in \mathcal{B}(H)$ is a contraction such that $\operatorname{dim} \mathcal{D}_{T}=\operatorname{dim} \mathcal{D}_{T^{*}}=$ 1 , and there exists no subspace of $H$ invariant by $T$ and such that its restriction therein is an isometry. Then there exists an extreme $b$ in the unit ball of $H^{\infty}$ such that $T$ is unitarily equivalent to $X_{b}$.

Proof. Since the proof is rather long, we divide it in several steps.

Step 1. Dilation of $T$. To find the required function $b$, we will develop a certain geometrical construction. Changing the order of the components in the range of the Julia operator yields a unitary operator mapping $H \oplus \mathcal{D}_{T^{*}}$ into $\mathcal{D}_{T} \oplus H$ according to the matrix $\left(\begin{array}{cc}D_{T} & -T^{*} \\ T & D_{T^{*}}\end{array}\right)$. We can extend this unitary to a unitary $W$ acting on the single enlarged space

$$
\mathfrak{H}=\cdots \oplus \mathcal{D}_{T^{*}} \oplus \mathcal{D}_{T^{*}} \oplus H \oplus \mathcal{D}_{T} \oplus \mathcal{D}_{T} \oplus \ldots
$$

that can be written as an bi-infinite operator matrix:

$$
W=\left(\begin{array}{llllllll}
\ddots & & & & & & & \\
& 1 & & & & & & \\
& & 1 & & & & & \\
& & & D_{T} & -T^{*} & & & \\
& & & \mathrm{~T} & D_{T^{*}} & & & \\
& & & & & 1 & & \\
& & & & & & 1 & \\
& & & & & & & \ddots
\end{array}\right)
$$

where the boxed entry corresponds to the central entry $T: H \rightarrow H$. If we write $\mathfrak{H}=\mathfrak{H}_{-} \oplus H \oplus \mathfrak{H}_{+}$, with

$$
\mathfrak{H}_{-}=\cdots \oplus \mathcal{D}_{T^{*}} \oplus \mathcal{D}_{T^{*}}, \quad \mathfrak{H}_{+}=\mathcal{D}_{T} \oplus \mathcal{D}_{T} \oplus \ldots,
$$

then $\mathfrak{H}_{-}$is invariant by $W$, which acts therein as translation to the left, while $\mathfrak{H}_{+}$is invariant by $W^{*}$, whose restriction is translation to the right. (This is a consequence of the fact that the 1 entries in the definition of $W$ are all located immediately above the main diagonal.)

Step 2. Two embeddings of $L^{2}$ into $\mathfrak{H}$. Take a unit vector $\epsilon_{-1}^{-}$in the $\mathcal{D}_{T^{*}}$ component of $\mathfrak{H}_{-}$which is mostly to the right, and define, for $n \in \mathbb{Z}, \epsilon_{n}^{-}=W^{-n-1} \epsilon_{-1}^{-}$. Since $\operatorname{dim} \mathcal{D}_{T^{*}}=1$, the family $\left(\epsilon_{n}^{-}\right)_{n \leq-1}$ forms an orthonormal basis of $\mathfrak{H}_{-}$. Moreover, the whole family $\left(\epsilon_{n}^{-}\right)_{n \in \mathbb{Z}}$ is an orthonormal set in $\mathfrak{H}$ (exercise!).

As $\left(e^{i n t}\right)_{n \in \mathbb{Z}}$ is an orthonormal basis in $L^{2}$, we may define $\omega_{-}: L^{2} \rightarrow \mathfrak{H}$ to be the unique isometry that satisfies $\omega_{-}\left(e^{i n t}\right)=\epsilon_{n}^{-}$for all $n \in \mathbb{Z}$. One checks easily that its image $\omega_{-}\left(L^{2}\right)$ is a reducing space for $W$, and $\omega_{-}^{*} W \omega_{-}=M_{e^{-i t}}$. The orthogonal projection onto $\omega_{-}\left(L^{2}\right)$ is $\omega_{-} \omega_{-}^{*}$, and it commutes with $W$. 
An analogous construction can be made for $\mathfrak{H}_{+}$. We obtain an orthonormal set $\left(\epsilon_{n}^{+}\right)_{n \in \mathbb{Z}}$ in $\mathfrak{H}$, such that $\left(\epsilon_{n}^{+}\right)_{n \geq 0}$ is a basis for $\mathfrak{H}_{+}$. Then $\omega_{+}: L^{2} \rightarrow \mathfrak{H}$ is the isometry that satisfies $\omega_{+}\left(e^{i n t}\right)=\epsilon_{n}^{+}$for all $n \in \mathbb{Z} ; \omega_{+}\left(L^{2}\right)$ is also a reducing space for $W, \omega_{+}^{*} W \omega_{+}=M_{e^{-i t}}$, and $\omega_{+} \omega_{+}^{*} W=W \omega_{+} \omega_{+}^{*}$.

Step 3. Finding $b$. Consider then the map $\omega_{-}^{*} \omega_{+}: L^{2} \rightarrow L^{2}$. We have, using the above remarks as well as the equalities $\omega_{+}^{*} \omega_{+}=\omega_{-}^{*} \omega_{-}=I_{L^{2}}$,

$$
\begin{aligned}
\omega_{-}^{*} \omega_{+} M_{e^{-i t}} & =\omega_{-}^{*} \omega_{+} \omega_{+}^{*} W \omega_{+}=\omega_{-}^{*} W \omega_{+} \omega_{+}^{*} \omega_{+}=\omega_{-}^{*} W \omega_{+} \\
& =\left(\omega_{-}^{*} \omega_{-}\right) \omega_{-}^{*} W \omega_{+}=\omega_{-}^{*} W \omega_{-} \omega_{-}^{*} \omega_{+}=M_{e^{-i t}} \omega_{-}^{*} \omega_{+} .
\end{aligned}
$$

So $\omega_{-}^{*} \omega_{+}$commutes with $M_{e^{-i t}}$; it follows that it commutes also with its inverse $M_{e^{i t}}$ (exercise!). We have noticed in the introduction that in this case we must have $\omega_{-}^{*} \omega_{+}=M_{b}$ for some function $b \in L^{\infty}$, and $\left\|\omega_{-}^{*} \omega_{+}\right\| \leq 1$ implies $\|b\|_{\infty} \leq 1$.

Now, $b=M_{b} \mathbf{1}=\omega_{-}^{*} \omega_{+} 1=\omega_{-}^{*} \epsilon_{0}^{+}$. Since $\epsilon_{0}^{+} \in \mathfrak{H}_{+} \perp \mathfrak{H}_{-}=\omega_{-}\left(\overline{H_{0}^{2}}\right)$, it follows that $\omega_{-}^{*} \epsilon_{0}^{+} \in H^{2}$. Thus $b \in L^{\infty} \cap H^{2}=H^{\infty}$, and we have found our candidate for the function in the unit ball of $H^{\infty}$. It remains now to check that it satisfies the required properties. As above, we will denote $\Delta=\left(1-|b|^{2}\right)^{1 / 2} \in L^{\infty}$.

Step 4. Constructing the unitary equivalence. Let us now note that the closed linear span $\omega_{+} L^{2} \vee \omega_{-} L^{2}$ equals $\mathfrak{H}$. Indeed, it reduces $W$ and contains $\mathfrak{H}_{+}$ and $\mathfrak{H}_{-}$; thus its orthogonal $Y$ has to be a reducing subspace of $W$ contained in $H$ (more precisely, in its embedding in $\mathfrak{H}$ ). From (8.1) it follows then that $W|Y=T| Y$, so $Y$ should be a subspace of $H$ invariant by $T$ and such that the restriction is isometric (even unitary!), which contradicts the hypothesis. Thus $Y=\{0\}$.

We define then a mapping $U: \omega_{+} L^{2} \vee \omega_{-} L^{2} \rightarrow L^{2} \oplus \overline{\Delta L^{2}}$ by

$$
U\left(\omega_{+} f_{+}+\omega_{-} f_{-}\right)=\left(f_{-}+b f_{+}\right) \oplus \Delta f_{+} .
$$

We have

$$
\begin{aligned}
\left\|\omega_{+} f_{+}+\omega_{-} f_{-}\right\|^{2} & =\left\|\omega_{+} f_{+}\right\|^{2}+\left\|\omega_{-} f_{-}\right\|^{2}+2 \Re\left\langle\omega_{+} f_{+}, \omega_{-} f_{-}\right\rangle \\
& =\left\|f_{+}\right\|_{2}^{2}+\left\|f_{-}\right\|_{2}^{2}+2 \Re\left\langle\omega_{-}^{*} \omega_{+} f_{+}, f_{-}\right\rangle_{2}=\left\|f_{+}\right\|_{2}^{2}+\left\|f_{-}\right\|_{2}^{2}+2 \Re\left\langle b f_{+}, f_{-}\right\rangle_{2},
\end{aligned}
$$

and

$$
\begin{aligned}
\left\|\left(f_{-}+b f_{+}\right) \oplus \Delta f_{+}\right\|^{2} & =\int\left|f_{-}+b f_{+}\right|^{2}+\int \Delta^{2}\left|f_{+}\right|^{2} \\
& =\int\left|f_{-}\right|^{2}+\left|b f_{+}\right|^{2}+2 \Re b f_{+} \bar{f}_{-}+\Delta^{2}\left|f_{+}\right|^{2} \\
& =\int\left|f_{-}\right|^{2}+\left|f_{+}\right|^{2}+2 \Re b f_{+} \bar{f}_{-},
\end{aligned}
$$

whence $U$ is an isometry, and it is easy to see that the image is dense. It can be extended to a unitary operator, that we will denote by the same letter,

$$
U: \mathfrak{H} \rightarrow L^{2} \oplus \overline{\Delta L^{2}} .
$$

The commutation relations satisfied by $\omega_{ \pm}$imply that $U W=\left(Z^{*} \oplus Z_{\Delta}^{*}\right) U$.

Step 5. Final checks. Now $U(\mathfrak{H})=U\left(\mathfrak{H}_{-}\right) \oplus U(H) \oplus U\left(\mathfrak{H}_{+}\right)$, and

$$
\begin{aligned}
& U\left(\mathfrak{H}_{-}\right)=U\left(\omega_{-}\left(\overline{H_{0}^{2}}\right)\right)=\left\{f_{-} \oplus 0: f_{-} \in \overline{H_{0}^{2}}=\overline{H_{0}^{2}} \oplus\{0\},\right. \\
& U\left(\mathfrak{H}_{+}\right)=U\left(\omega_{+}\left(H^{2}\right)\right)=\left\{b f_{+} \oplus \Delta f_{+}: f_{+} \in H^{2}\right\},
\end{aligned}
$$


So

$$
\begin{aligned}
U(H) & =\left(L^{2} \oplus \overline{\Delta L^{2}}\right) \ominus\left(\left(\overline{H_{0}^{2}} \oplus\{0\}\right) \oplus\left(\left\{b f_{+} \oplus \Delta f_{+}: f_{+} \in H^{2}\right\}\right)\right) \\
& =\left(H^{2} \oplus \overline{\Delta L^{2}}\right) \ominus\left(\left\{b f_{+} \oplus \Delta f_{+}: f_{+} \in H^{2}\right\}\right) .
\end{aligned}
$$

As shown by (8.1), $T$ can be viewed as the compression of $W$ to $H$, so it is unitarily equivalent through $U$ to the compression of $Z^{*} \oplus Z_{\Delta}^{*}$ to $U(H)$. This last is easily seen to be the restriction of $S^{*} \oplus Z_{\Delta}^{*}$ to $\left(H^{2} \oplus \overline{\Delta L^{2}}\right) \ominus\left(\left\{b f_{+} \oplus \Delta f_{+}: f_{+} \in H^{2}\right\}\right)$.

We are very close to the end: Theorem 5.8 would end the proof, provided we could replace in the formula for $U(H)$ the space $H^{2} \oplus \overline{\Delta L^{2}}$ with $H^{2} \oplus \overline{\Delta H^{2}}$ and thus $Z_{\Delta}$ with $V_{\Delta}$. But the space

$$
Y:=\left(H^{2} \oplus \overline{\Delta L^{2}}\right) \ominus\left(H^{2} \oplus \overline{\Delta H^{2}}\right)=\{0\} \oplus\left(\overline{\Delta L^{2}} \ominus \overline{\Delta H^{2}}\right)
$$

is invariant with respect to $S^{*} \oplus Z_{2}^{*}$, which acts on it isometrically. By assumption, we must have $Y=\{0\}$, which means that $H^{2} \oplus \overline{\Delta L^{2}}=H^{2} \oplus \overline{\Delta H^{2}}$; this finishes the proof.

Exercise 8.2. Show that Theorem 8.1 implies Theorem 3.2

\section{Further REAding}

We discuss in this section some directions in which the study of de BrangesRovnyak spaces has developed.

The model spaces $\mathbf{K}_{u}$ have no nonconstant multipliers. However, the theory of multipliers is interesting for the case of de Branges-Rovnyak spaces corresponding to nonextreme $b$; see references [16, 17, 18.

Integral representations of de Branges-Rovnyak spaces appear in 4], and have further been developed in [21, 12, 13. The last paper is used in [5] to obtain weighted norm inequalities for functions in de Branges-Rovnyak spaces.

The connection between de Branges-Rovnyak and Dirichlet spaces is exploited in [8, 9]; that between de Branges-Rovnyak spaces and composition operators in 15 .

Finally, it is natural from many points of view (including that of model spaces) to consider also matrix or operator valued de Branges-Rovnyak spaces. These have already been introduced in [4]; see [2, 3, for some recent developments.

\section{ACKNOWLEDGEMENTS}

This work was partially supported by a grant of the Romanian National Authority for Scientific Research, CNCS UEFISCDI, project number PN-II-ID-PCE2011-3-0119.

This work will appear in the Proceedings Volume of the CRM Conference Invariant Subspaces of the Shift Operator, August 26-30, 2013. The author is grateful to the organizers of the Conference for the support provided. 


\section{REFERENCES}

[1] N. Aronszajn, Theory of reproducing kernels, Trans. AMS 68 (1950), 337-404.

[2] J.A. Ball, V. Bolotnikov, S. ter Horst, Interpolation in de Branges-Rovnyak spaces, Proc. Amer. Math. Soc. 139 (2011), 609-618.

[3] J.A. Ball, V. Bolotnikov, S. ter Horst, Abstract interpolation in vector-valued de BrangesRovnyak spaces, Integral Equations Operator Theory 70 (2011), 227-263.

[4] J.A. Ball, Th.L. Kriete, Operator-valued Nevanlinna-Pick kernels and the functional models for contraction operators, Integral Equations Operator Theory 10 (1987), 17-61.

[5] A. Baranov, E. Fricain, J. Mashreghi, Weighted norm inequalities for de Branges-Rovnyak spaces and their applications, Amer. J. Math. 132 (2010), 125-155.

[6] L. de Branges, J. Rovnyak, Square Summable Power Series, Holt, Rinehart and Winston, 1966.

[7] L. de Branges, J. Rovnyak, Canonical models in quantum scattering theory, in Perturbation Theory and its Applications in Quantum Mechanics, ed. by C.H. Wilcox, Wiley, 1966, 295392.

[8] N. Chevrot, D. Guillot, Th. Ransford, De Branges-Rovnyak spaces and Dirichlet spaces, J. Funct. Anal. 259 (2010), 2366-2383.

[9] C. Costara, Th. Ransford, Which de Branges-Rovnyak spaces are Dirichlet spaces (and vice versa)?, J. Funct. Anal. 265 (2013), 3204-3218.

[10] R.G. Douglas, On majorization, factorization, and range inclusion of operators on Hilbert space, Proc. AMS 17 (1966), 413-415.

[11] E. Fricain, Bases of reproducing kernels in de Branges spaces, J. Funct. Anal. 226 (2005), 373-405.

[12] E. Fricain, J. Mashreghi, Boundary behavior of functions in the de Branges-Rovnyak spaces, Complex Anal. Oper. Theory 2 (2008), 87-97.

[13] E. Fricain, J. Mashreghi, Integral representation of the $n$-th derivative in de Branges-Rovnyak spaces and the norm convergence of its reproducing kernel, Ann. Inst. Fourier (Grenoble) 58 (2008), 2113-2135.

[14] Emmanuel Fricain, Javad Mashreghi: Theory of $\mathcal{H}(b)$ Spaces, vol. I and II, New Monographs in Mathematics, Cambridge University Press, to appear.

[15] M.T. Jury, Reproducing kernels, de Branges-Rovnyak spaces, and norms of weighted composition operators, Proc. Amer. Math. Soc. 135 (2007), 3669-3675.

[16] B.A. Lotto, D. Sarason, Multiplicative structure of de Branges's spaces, Rev. Mat. Iberoamericana 7 (1991), 183-220.

[17] B.A. Lotto, D. Sarason, Multipliers of de Branges-Rovnyak spaces, Indiana Univ. Math. J. 42 (1993), 907-920.

[18] B.A. Lotto, D. Sarason, Multipliers of de Branges-Rovnyak spaces, II, in Harmonic Analysis and Hypergroups, Delhi, 1995, 51-58.

[19] N.K. Nikolski: Operators, Functions, and Systems: An Easy Reading, AMS, 2002.

[20] N.K. Nikolski, V.I. Vasyunin, Notes on two function models, in The Bieberbach conjecture (West Lafayette, Ind., 1985), , Math. Surveys Monogr., 21, Amer. Math. Soc., Providence, RI, 1986, 113-141.

[21] A.G. Poltoratskii, Boundary behavior of pseudocontinuable functions, Algebra i Analiz 5 (1993), 189-210, translation in St. Petersburg Math. J. 5 (1994), no. 2, 389-406.

[22] D. Sarason, Sub-Hardy Hilbert Spaces in the Unit Disk, John Wiley, 1994.

[23] D. Sarason, Local Dirichlet spaces as de Branges-Rovnyak spaces, Proc. Amer. Math. Soc. 125 (1997), 2133-2139.

[24] B. Sz-Nagy, C. Foias, H. Bercovici, L. Kérchy: Harmonic Analysis of Operators on Hilbert space, Springer, 2010.

Institute of Mathematics of the Romanian Academy, P.O. Box 1-764, Bucharest 014700, ROMANIA

E-mail address: Dan.Timotin@imar.ro 